\title{
Materialaspekte bei der thermischen Energiespeicherung in Flüssigsalz
}

\author{
Alexander Bonk, ${ }^{1}$ Claudia Martin, ${ }^{1}$ Thomas Bauer ${ }^{2}$ \\ ${ }^{1}$ German Aerospace Center (DLR), Institute of Technical Thermodynamics, 70569 Stuttgart, Germany \\ ${ }^{2}$ German Aerospace Center (DLR), Institute of Technical Thermodynamics, 51147 Cologne, Germany,
}

Thermische Energiespeicher auf Basis von Flüssigsalzen spielen eine wichtige Rolle bei der Zwischenspeicherung von überschüssiger thermischer oder elektrischer Energie. Im Falle der Sensiblen und Temperaturschicht-Salzspeicher liegt der anwendbare Temperaturbereich zwischen der Erstarrungstemperatur des Salzes, typischerweise $\quad \mathrm{NaNO}_{3}-\mathrm{KNO}_{3}$-Mischungen, und dessen oberer Zersetzungstemperatur. Ein umfassendes Verständnis für die Salzchemie ist in beiden Speichern unabdingbar für die Auslegung und den Betrieb des Speichers selbst. Dabei spielen, im Falle der Sensiblen Speicher, vor allem Zersetzungsreaktionen der Nitratsalze bei hohen Temperaturen eine Rolle, da diese einen signifikanten Einfluss auf die thermischen Eigenschaften, und damit den Nutzbereich, haben. Bei Temperaturschichtspeichern, die Füllermaterialien auf Mineralbasis nutzen um teures Salzvolumen zu verdrängen, gesellen sich zu den Salz-Fragestellungen noch Herausforderungen bezüglich der Beständigkeit der eingesetzten Füllermaterialien gegenüber dem Salz.

Die hier vorgestellte Arbeit befasst sich umfassend mit Materialaspekten bei der thermischen Energiespeicherung und beantwortet gezielt Fragestellungen über die Degradationsmechanismen von Nitratsalzen und der Füllermaterialien. Experimentelle Daten werden dabei über die Auslagerung von Nitrat-Salzen in einer Mehrfach-Messkammer unter Realbedingungen und anschließender Analyse erzielt. Die Abhängigkeit der Salzzersetzung von Temperatur und Gasatmosphären wird dabei in den Vordergrund gestellt. Die Resultate zeigen zum Beispiel, dass die Gasatmosphären einen signifikanten Einfluss auf das Nitrat-Nitrit Gleichgewicht haben, welches wiederrum drastisch den Schmelzpunkt beeinflusst.

Im Falle der Temperaturschichtspeicher wird eine Auswahl geeigneter Füllermateralien auf Basis experimenteller Daten getroffen und präsentiert. Dabei steht vor allem die Phasenstabilität der typischerweise mineralischen Füller, sowie die Entwicklung der Salzchemie im Vordergrund. 